\title{
Influence of Carbon Nanotubes Dispersion on Thermal Properties of Copper-Carbon Nanotubes (CNTs) Composite Materials
}

\author{
Guy-Marie Vallet ${ }^{1,3, *}$, Michel Dunand ${ }^{1}, J^{\prime}$ ean-Francois Silvain ${ }^{2}$ \\ ${ }^{1}$ Labinal Power Systems, France \\ ${ }^{2}$ Centre National De La Recherche Scientifique (CNRS), Institute of Condensed Matter Chemistry of Bordeaux (ICMCB), France \\ ${ }^{3}$ Institute of Condensed Matter Chemistry of Bordeaux (ICMCB), University of Bordeaux, France
}

Copyright $(C 2015$ by authors, all rights reserved. Authors agree that this article remains permanently open access under the terms of the Creative Commons Attribution License 4.0 International License

\begin{abstract}
In this present work, two methods for dispersing carbon nanotubes into the copper matrix were tested: a Solid Route process where CNTs are simply mixed with the copper powder and a Liquid Route process where CNTs are dispersed in a copper salt solution and then mixed with the metallic copper powder. Powders are sintered by uni-axial hot pressing process under vacuum atmosphere at $650^{\circ} \mathrm{C}$ and thermal conductivities of composite materials were measured using the laser flash method. Results are compared with a theoretical model of Nan et al. which enables to predict the thermal conductivity of materials containing CNTs. Comparison of experimental and theoretical results tends to prove that CNTs are 2D-randomly dispersed in a plane perpendicular to the pressing direction during uni-axial hot pressing process. Moreover, an increase of $+7 \%$ of the thermal conductivity is shown for the composite material containing 1 vol. $\%$ of CNTs into the copper matrix.
\end{abstract}

Keywords Composite Materials, Powder Metallurgy, Thermal Conductivity, Thermal Properties

\section{Introduction}

Since their discovery in 1991 by Iijima ${ }^{[1]}$, carbon nanotubes are intensively studied. Theory predicts huge mechanical ( $\left.E_{\text {Young }}=1 \mathrm{TPa}\right)$, thermal (3000 to $\left.6000 \mathrm{~W} \cdot \mathrm{m}^{-1} \cdot \mathrm{K}^{-1}\right)$ and electrical $\left(10^{7}\right.$ to $\left.10^{9} \mathrm{~A} . \mathrm{cm}^{-2}\right)$ properties to carbon nanotubes that makes them interesting as reinforcements in composite materials. Several publications already exist on the addition of carbon nanotubes into a polymer matrix showing an enhancement of physical properties. The resulting composite material possesses better mechanical ${ }^{[2][3]}$, thermal ${ }^{[4]}$ and electrical ${ }^{[5] 6][7]}$ properties than the pure matrix. However just a few limited studies exist in the literature
[8][9][10] concerning the use of metal-matrix due to the extreme conditions of processing for the composite. Furthermore, some difficulties are still a critical issue like the dispersion of the CNTs inside the matrix and the interface resistance, i.e. thermal, mechanical, between the CNT and the metal-matrix. Despite that, the perspective of obtaining better properties than pure metallic matrix encourages scientists to persevere in this way. As shown in the literature, the thermal properties of Cu-CNTs composite material depend i) on the quality of the dispersion of carbon nanotubes into the matrix and ii) on the interface thermal resistance between the reinforcement and the matrix. Cho et al. ${ }^{[11]}$ prepared materials containing Multi Wall Carbon Nanotubes (MWCNT) by spark plasma sintering and shows a 3\% increase of the thermal conductivity of the composite materials, compared with pure copper, for a volume fraction of $1 \%$ nanotubes. Chu et al ${ }^{[12]}$ ${ }^{[13]}$ fabricated $\mathrm{Cu}-\mathrm{CNTs}$ materials with a novel technique for dispersing carbon nanotubes into the matrix. Despite a relative good dispersion of the reinforcement, the thermal conductivity of the composite materials was lower than the copper reference. Chen ${ }^{[14]}$ proposed the concept of electro codeposition for the fabrication of $\mathrm{Cu} / \mathrm{CNTs}$ composite materials. In a first step, SWCNTs are functionalized in order to be positively charged. In a second step, SWCNTs are placed in a bath containing copper ions and an $\mathrm{H}_{2} \mathrm{SO}_{4}$ electrolyte. A current passes through the solution and a thin layer of $\mathrm{Cu}-\mathrm{CNTs}$ is deposited on the electrode. Thermal characterization shows that the material exhibits a thermal conductivity of $640 \mathrm{~W} \cdot \mathrm{m}^{-1} \cdot \mathrm{K}^{-1}$ which is a value $60 \%$ higher than pure copper. Quantify the dispersion of nanotubes into a matrix is mandatory for the understanding of final results. SEM and TEM analysis may give some local information. Nevertheless, microscopic observations are not sufficient to quantify the dispersion at macroscopic scale. The use of a theoretical model, using an effective medium approach like the model of Maxwell-Garnett modified by Nan et al. ${ }^{[15]}$ for composite materials containing CNTs, could be a solution. 
This model enables to predict the thermal conductivity of materials if and only if carbon nanotubes are well-dispersed inside the matrix and if the CNT concentration is smaller than the percolation threshold. This model takes into account the conductivity and the aspect ratio of the tube, the conductivity of the matrix and the interfacial thermal resistance between CNTs and the matrix. Nowadays, it is well known that the interface between the components remains one of the major problem areas in which copper properties can be improved. In 2003, Huxtable et al. ${ }^{[16]}$ determined the value of the interfacial thermal resistance of a carbon nanotube suspended in surfactant micelles. A value of $\mathrm{R}_{\mathrm{th}}=8.310^{-8} \mathrm{~m}^{2} . \mathrm{K} . \mathrm{W}^{-1}$ was calculated and is now used in most publications. In this study, using powder metallurgy process, MWCNT have been dispersed inside a dendritic copper powder using two different methods that we have called "Solid Route" and "Liquid Route". After densification using uni-axial hot pressing, the dispersion of CNTs inside the matrix is quantified through the use of the Maxwell-Garnett model modified by Nan et al. Thermal conductivities parallel and perpendicular to the pressing direction are measured using conventional laser flash method.

\section{Materials and Methods}

Multi-walled carbon nanotubes (NC7000), with an average diameter of $9.5 \mathrm{~nm}$ and an average length of $1.5 \mu \mathrm{m}$ have been purchased from Nanocyl ${ }^{\mathrm{TM}}$ Company to conduct the following experiments. Compared to other works in the literature ${ }^{[17][18]}$, the MWCNTs used in this work have not been acid treated in order to not attach oxygen groups onto the CNTs surface. Dendritic shape copper powders (20-30 $\mu \mathrm{m}$ mean grain size) from Ecka Granules Company were used without any additional purification. Two routes of dispersion for carbon nanotubes have been conducted. A "Solid Route" (SR) where carbon nanotubes were simply mixed 2 hours in a planetary mixer with the metallic copper powder and a "Liquid Route" (LR) where the use of chemical species and physical methods allows a good dispersion of nanotubes into the matrix ${ }^{[191-[24]}([\mathrm{CNTs}] /[\mathrm{Cu}$ precursor $]=0.05$ ). Then the $\mathrm{Cu} @$ CNTs powders are uni-axial hot pressed at a temperature of $650^{\circ} \mathrm{C}$ during 20 minutes in a vacuum atmosphere. For each route, several composite materials (pellet) were fabricated with a diameter of $6 \mathrm{~mm}$ diameter and a thickness of $3.2 \mathrm{~mm}$. Thermal diffusivities are measured at $70^{\circ} \mathrm{C}$ with laser flash method on LFA 457 device from Netzsch Company. Thermal diffusivities were converted into thermal conductivities with the following formula: $\lambda=\alpha \rho C_{p}$ where $\lambda$ is the thermal conductivity $\left(\mathrm{W} \cdot \mathrm{m}^{-1} \cdot \mathrm{K}^{-1}\right), \alpha$ is the thermal diffusivity $\left(\mathrm{m}^{2} \cdot \mathrm{s}^{-1}\right), \mathrm{C}_{\mathrm{p}}$ is the heat capacity of the composite material at constant pressure $\left(\mathrm{J} \cdot \mathrm{kg}^{-1} \cdot \mathrm{K}^{-1}\right)$ at $70^{\circ} \mathrm{C}$ and $\rho$ is the density $\left(\mathrm{kg} \cdot \mathrm{m}^{-3}\right)$ of the composite material. $\rho$ was measured by the geometrical method. $\mathrm{C}_{\mathrm{p}}$ of copper and $\mathrm{C}_{\mathrm{p}}$ of carbon nanotubes have been measured by Differential Scanning Calorimetry (DSC) on a
8000/8500 Perkin-Elmer apparatus. $C_{p}$ of composite material was calculated with a simple rule of mixture from raw materials. Scanning Electron Microscopy analysis was performed in two modes (Secondary Electron (SE) and Back-Scattered Electron (BSE)) with a Tescan Vega II from Eloise France Company. Pellet micrographs of the $\mathrm{Cu}-\mathrm{CNTs}$ composite materials were obtained on a Leica microscope with VZ80 RC lens and a DVM 2500 digital camera from Leica Microsystems Company.

\section{Results and Discussion}

\subsection{Thermal Characterization}

$\mathrm{Cu}$ and $\mathrm{Cu}-\mathrm{CNTs}$ pellets composite materials produced by the use of the Solid and Liquid Routes processes have been analyzed by thermal diffusivity measurements and then converted into thermal conductivities as shown in Table 1 . For each carbon nanotube content the thermal conductivity is an average of three different pellets and measurements are done in the direction parallel to the axis of compression.

Table 1. Thermal conductivities of Cu-CNTs composite materials obtained by Solid Route and Liquid Route.

\begin{tabular}{|c|c|c|c|c|}
\hline \multirow{2}{*}{$\begin{array}{c}\text { Volume } \\
\text { fraction of } \\
\begin{array}{c}\text { CNTs } \\
(\%)\end{array}\end{array}$} & $\begin{array}{c}\text { Thermal } \\
\text { conductivity } \\
\left(\mathrm{W} \cdot \mathrm{m}^{-1} \cdot \mathrm{K}^{-1}\right)\end{array}$ & $\begin{array}{c}\text { Relative } \\
\text { density } \\
(\%)\end{array}$ & $\begin{array}{c}\text { Thermal } \\
\text { conductivity } \\
\left(\mathrm{W} \cdot \mathrm{m}^{-1} \cdot \mathrm{K}^{-1}\right)\end{array}$ & $\begin{array}{c}\text { Relative } \\
\text { density } \\
(\%)\end{array}$ \\
\hline 0 & 375 & 98 & 375 & 98 \\
\hline 0.5 & 372 & 97 & 373 & 98 \\
\hline 1.0 & 339 & 95 & 364 & 98 \\
\hline 1.5 & 336 & 97 & 362 & 98 \\
\hline 3.0 & 272 & 96 & 337 & 99 \\
\hline
\end{tabular}

\subsubsection{Copper Materials Fabricated with a Powder Metallurgy Process}

Table 1 shows pure copper pellets properties, fabricated using powder metallurgy method and having an average thermal conductivity of $375 \mathrm{~W} \cdot \mathrm{m}^{-1} \cdot \mathrm{K}^{-1}$. This thermal conductivity value is slightly lower than the thermal conductivity of bulk copper reference material known as 400 $\mathrm{W} \cdot \mathrm{m}^{-1} \cdot \mathrm{K}^{-1}$. This lower thermal conductivity value can be attributed to the microstructure (smaller grain size) of the powder metallurgy materials and of the $2 \%$ remaining porosity measured. Moreover, Cernushi et al. ${ }^{[23]}$ have shown that, the graphite layer, which is sputtered on both sample faces before laser flash measurement and which prevents the laser reflection, has a negative effect on the thermal conductivity value measured. Using the Voigt-Reuss model for in series material, the total thermal conductivity can be calculated for the three-layers material $(\mathrm{C} /(\mathrm{Cu}-\mathrm{CNT}) / \mathrm{C})$. Thickness of the graphite spray onto the surface was measured by optical profilometry. Results show that the graphite layer is non-homogeneously sputtered onto the surface; its thickness varying from $2 \mu \mathrm{m}$ to $12 \mu \mathrm{m}$. Moreover, the thermal conductivity of the graphite spray is assumed to 
be close to $24 \mathrm{~W} \cdot \mathrm{m}^{-1} \cdot \mathrm{K}^{-1}$ as mentioned by Cernushi et al. ${ }^{[22]}$. Considering the thermal conductivity of pure copper equal to $400 \mathrm{~W} \cdot \mathrm{m}^{-1} \cdot \mathrm{K}^{-1}$, Figure 1 shows the calculated thermal conductivity of the three-layers sample for different graphite layers thickness. From Figure 1, it is obvious that the thickness of graphite layer must be carefully controlled if we want to compare the thermal conductivities values of different composite materials. If we consider that the graphite layer on the top and on the bottom face of the pure copper pellet ranges from $0 \mu \mathrm{m}$ to $12 \mu \mathrm{m}$, the average thermal conductivity of the pellet calculated -using the Vogt-Reuss model- is equal to $378 \mathrm{~W} \cdot \mathrm{m}^{-1} \cdot \mathrm{K}^{-1}$. This calculated thermal conductivity value is very close to the thermal conductivity measured on pure copper pellet $(375$ $\mathrm{W} \cdot \mathrm{m}^{-1} \cdot \mathrm{K}^{-1}$ ) obtained by powder metallurgy process and measured by the laser flash method. Consequently, if we consider that the technique for sputtering graphite onto the surface of the composite materials is repeatable, we can consider that the measured thermal conductivities of the three-layers materials $\mathrm{C} /(\mathrm{Cu}-\mathrm{CNT}) / \mathrm{C}$ are $6 \%$ lowered than the real materials $(\mathrm{Cu}-\mathrm{CNT})$ without graphite layers.

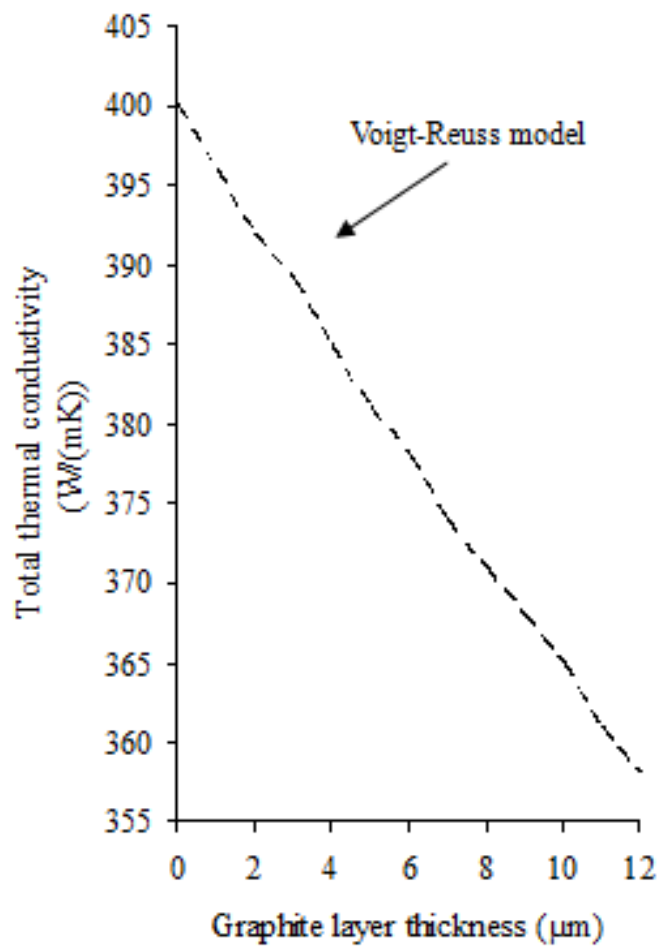

Figure 1. Evolution of the total thermal conductivity as a function of the graphite layer thickness

\subsubsection{Cu-CNTs Composite Materials Fabricated with a Powder Metallurgy Process}

According to Table 1, with a carbon nanotubes content of 0.5 vol. $\%$, no difference is observed between the Solid and the Liquid Routes whereas a different thermal conductivity is measured for a volume fraction of carbon nanotubes higher than 0.5 vol. \% of CNTs. Materials produced by Solid Route present a decrease of the thermal conductivity higher than the materials produced by the use of the Liquid Route. This thermal conductivity difference between the two fabrication processes may be attributed to the formation of carbon nanotubes clusters inside the metal matrix material.

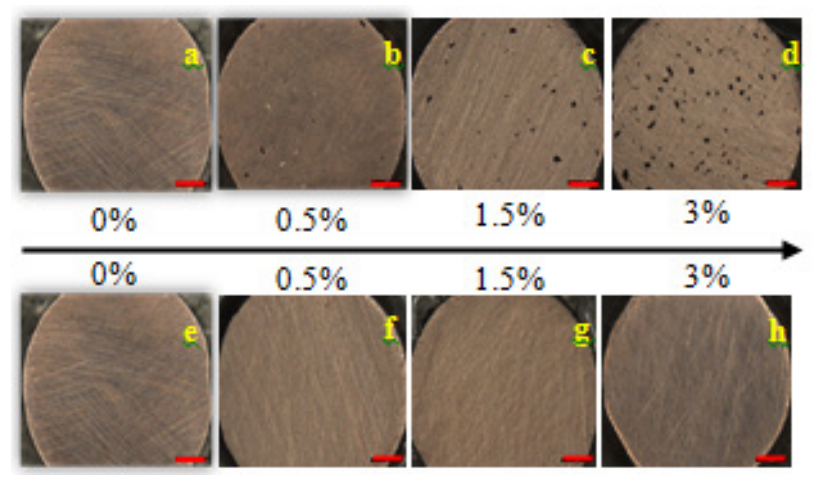

Figure 2. Surface sample of Cu-CNTs materials produced a)b)c)d) by Solid Route process and e)f)g)h) by Liquid Route process (Scale $1 \mathrm{~mm}$ )

Figure 2 shows typical optical micrographs obtained for $\mathrm{Cu}-\mathrm{CNT}$ materials produced by SR and LR. Dark spots can be observed in materials produced by Solid Route which is not the case for $\mathrm{Cu}-\mathrm{CNTs}$ pellets obtained by the Liquid Route process. As soon as carbon nanotubes content increase in the matrix with the SR process, the quantity of dark spots increase also.
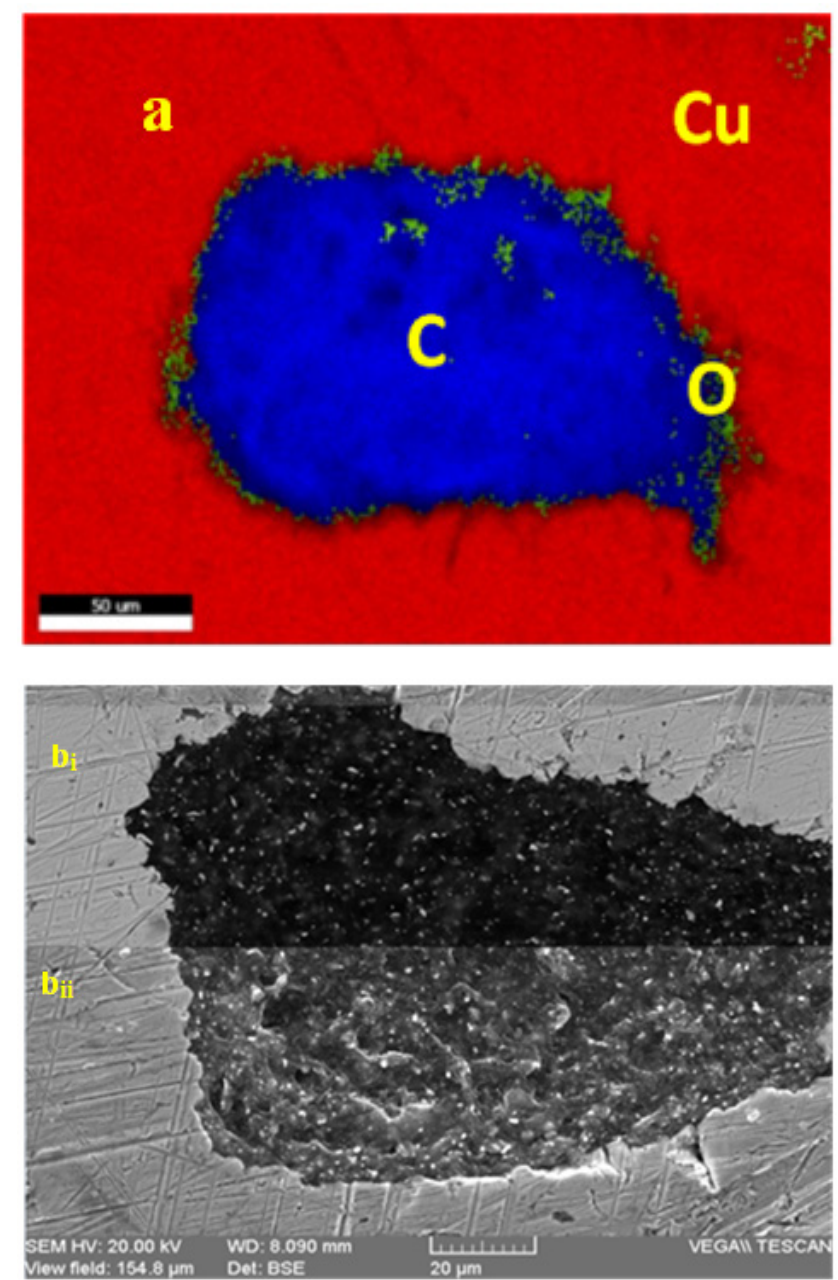


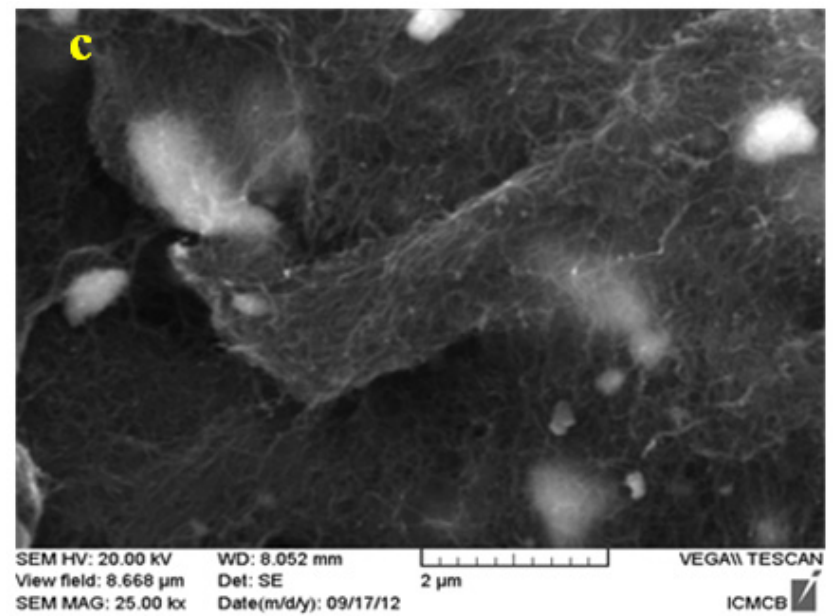

Figure 3. a) Chemical analysis of a porosity on the surface of $\mathrm{Cu}-\mathrm{CNTs}$ sample produced by Solid Route, Scanning Electron Micrographs in $\left(b_{i} \& c\right)$ secondary electron mode and $\left(b_{i i}\right)$ back-scattered electron mode showing carbon nanotubes into pore

\subsection{Maxwell-Garnett Model Modified by Nan et al}

The model of Maxwell-Garnett modified by Nan et al. enables to predict the thermal conductivity of material containing carbon nanotubes, if and only if, carbon nanotubes are well-dispersed into the matrix. Nevertheless, according to Nan et al., the model will underestimate the effective thermal conductivity of the composite material above the percolation threshold especially when the ratio $\mathrm{K}_{\mathrm{CNT}} / \mathrm{K}_{\text {Matrix }}$ is greater than $100^{[25]}$. The percolation threshold value $\left(\Phi_{\mathrm{p}}\right)$ of CNTs uniformly dispersed into a copper matrix was calculated using two different models explained by Berhan et al. ${ }^{[26]}$. The percolation threshold is estimated to be between 0.2 vol. $\%$ of CNTs $<\Phi_{\mathrm{p}}<1.5$ vol. $\%$ of CNTs. Therefore, under a value of 1.5 vol. $\%$ of CNTs, the model will be considered as valid. Beyond the percolation threshold, carbon nanotubes are not considered anymore as uniformly dispersed reinforcements and the model is no more valid. The measured thermal conductivities of fabricated materials are therefore compared with the effective thermal conductivity predicted by the model. This comparison is indeed linked with the dispersion degree of the CNTs inside the copper matrix. The effective thermal conductivity of the composite is expressed as (1) and (2) and detailed equations are explained in Appendix.

Two different values of $\left\langle\cos ^{2} \theta\right\rangle$ have been defined by Nan et al. representing two limiting cases (Figure 4). The first case, where $\left\langle\cos ^{2} \theta\right\rangle=1 / 3$, represents a random orientation of the CNT inside the $\mathrm{Cu}$ matrix and the second case, where $\left\langle\cos ^{2} \theta\right\rangle=1$, represents an orientation of CNT parallel to the $X_{3}^{*}$ direction. This model enables to calculate the resulting effective thermal conductivity along the $K_{11}^{*}, K_{22}^{*}$ and $K_{33}^{*}$ directions of the material. In the case of $\left\langle\cos ^{2} \theta\right\rangle=1 / 3, K_{11}^{*}=K_{22}^{*}=K_{33}^{*}=K^{*}$. For the
Maxwell-Garnett model modified by Nan et al. we assume that the thermal conductivity along the tube direction is about $3000 \mathrm{~W} \cdot \mathrm{m}^{-1} \cdot \mathrm{K}^{-1}$ which is a value commonly used in literature for MWCNT. Experimental results and theoretical model are compared on Figure 5 and Figure 6.

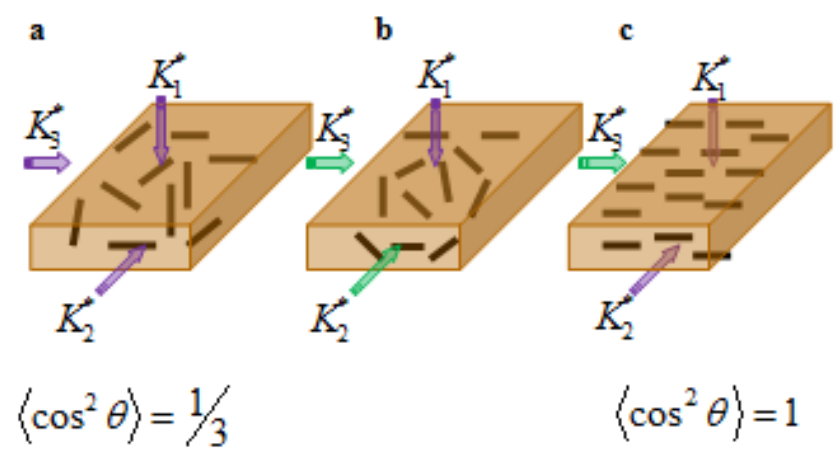

Figure 4. Cu-CNTs material for a) a random orientation of CNTs $\left(\left\langle\cos ^{2} \theta\right\rangle=1 / 3\right)$, b) CNTs 2D-randomly orientated in a plane perpendicular to the axis of compression and c) CNTs orientated along one direction $\left(\left\langle\cos ^{2} \theta\right\rangle=1\right)$

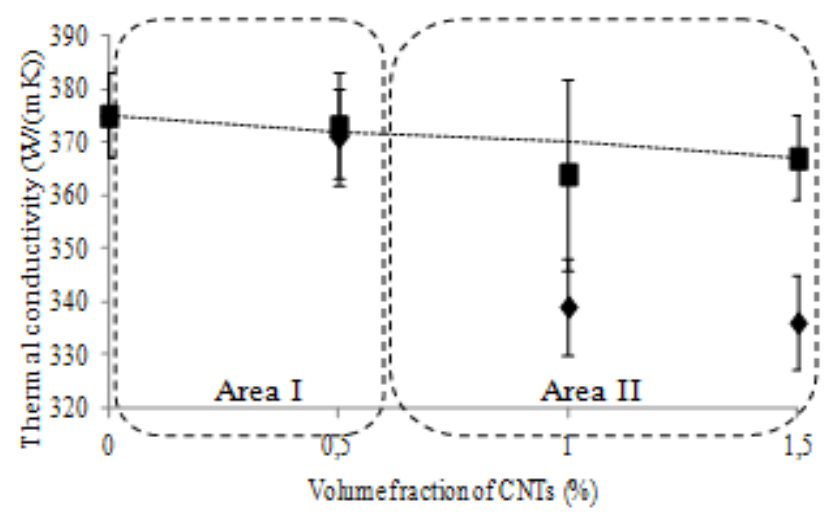

- Solid Route (Exp.) D Liquid Route (Exp.) -..-‥-- $\mathrm{K}^{*}$ (calculated)

Figure 5. Measured thermal conductivities of $\mathrm{Cu}-\mathrm{CNTs}$ composite materials parallel to the pressing direction and predicted thermal conductivities $\left(K^{*}\right)$ for $\left\langle\cos ^{2} \theta\right\rangle=1 / 3$

For a carbon nanotube concentration under the percolation threshold two areas can be defined. In the "Area I", where the carbon nanotubes content is lower than 0.5 vol. \%, the composite materials fabricated with the SR and the LR processes are in good correlation with the model of Maxwell-Garnett. Indeed, the CNTs clusters induced by the use of the SR process are not sufficient to affect the thermal conductivity of the material. In the "Area II", where the concentration of nanotubes is between 0.5 vol. \% and 1.5 vol. \%, only the thermal conductivities of Cu-CNTs materials fabricated using LR process are in good correlation with the MG model. For materials fabricated using SR process, the experimental values and the prediction from the model are tending to decrease when the volume fraction of reinforcements increases. These observations confirm that the use of a theoretical model, which enables to predict the thermal conductivity, can be a way to determine the quality of the carbon nanotubes dispersion into a metal-matrix 
composite material. Moreover, the thermal conductivity values for the LR process are in good agreement with the model when $\left\langle\cos ^{2} \theta\right\rangle$ equal to $1 / 3$ (random orientation of the CNT). Therefore, before concluding to a 3D-randomly orientation of the nanotubes into the matrix it is important to measure the thermal conductivity of the $\mathrm{Cu}$-CNTs composite materials in the direction perpendicular to the axis of compression. Indeed, the uni-axial hot pressing process could lead to an orientation of carbon reinforcements in a plane perpendicular to the axis of compression -as already observed for Copper-Carbon Fibers and Aluminum-Carbon Fibers composites materials- resulting to an anisotropy of the thermal conductivity values for the $\mathrm{Cu}-\mathrm{C}$ based composite materials. For materials fabricated with the LR process, Figure 6 shows the experimental values of thermal conductivity in the parallel (Liquid Route //) and in the perpendicular (Liquid Route $\perp$ ) directions compared to the axis of compression. The predictions of MG model are also presented for a value of $\left\langle\cos ^{2} \theta\right\rangle$ equal to 1 which corresponds to a perfect alignment of carbon nanotubes along one direction in the copper matrix (Figure 4). As it can be shown, $\mathrm{Cu}-\mathrm{CNTs}$ materials present anisotropic thermal conductivities since the values in the parallel direction are different of the thermal conductivity values in the perpendicular direction. These calculated values confirm that carbon nanotubes are not 3D-randomly dispersed in the material. Moreover, thermal conductivity values in the perpendicular direction are $7 \%$ higher than the copper reference showing a composite effect between the matrix and the reinforcements. This observation confirms that carbon nanotubes are able to enhance the thermal properties compared to a pure copper matrix. Note that this increase of thermal conductivity is higher than thermal conductivity of $\mathrm{Cu}-\mathrm{CNTs}$ presented in others works. Indeed, most part of the articles show thermal conductivity measurements with acid-treated CNTs. But the acidification of the reinforcements leads to a partial loss of carbon nanotube properties. Moreover, the increase of $7 \%$ of the thermal conductivity, in this work, is far away compared to the value of $640 \mathrm{~W} \cdot \mathrm{m}^{-1} \cdot \mathrm{K}^{-1}$ presented by Chen ${ }^{[14]}$. This discrepancy is probably due to the fact that we use multiwalled carbon nanotubes which present thermal properties lower than singlewalled carbon nanotubes.

The theoretical predictions from the MG model are in good agreement with the experimental values in the $K_{11}^{*}$ direction meaning that carbon nanotubes are orientated in a plane perpendicular to the axis of compression. Therefore, no correlation is observed between the MG model and the thermal conductivity values in the $K_{33}^{*}$ direction. Indeed, considering a value of $\left\langle\cos ^{2} \theta\right\rangle$ equal to 1 means that carbon nanotubes are perfectly aligned in the $K_{33}^{*}$ direction. Taking into account our experimental analyses, it is obvious, that during the uni-axial hot pressing process, the reinforcements are not aligned in one direction but probably 2D-randomly dispersed in a plane perpendicular to the axis of compression.

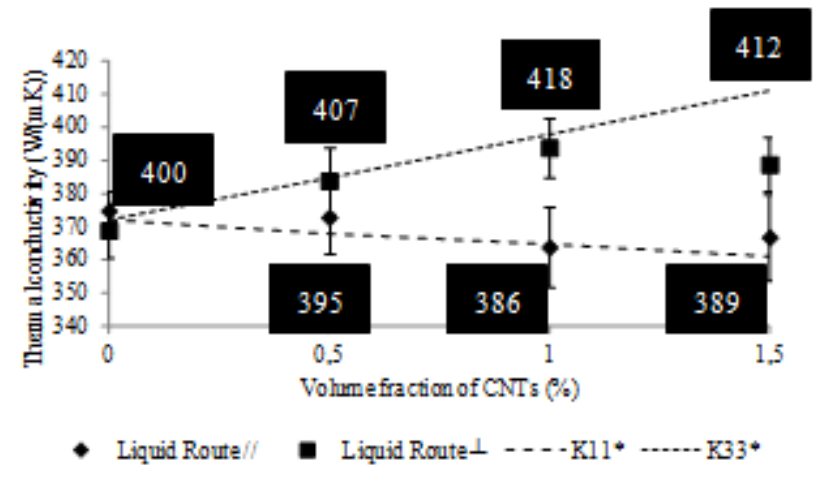

Figure 6. Thermal conductivity of Cu-CNTs composite materials and predicted results ( $K_{11}^{*}$ and $K_{33}^{*}$ ) for $\left\langle\cos ^{2} \theta\right\rangle=1$. Calculated results in the dark boxes may refer to materials without graphite layer sputtered onto the surface

Due to the fact that previous work on Copper-Carbon Fibers have shown that, after hot pressing, carbon fibers are randomly orientated in a plane perpendicular to the axis of compression and that predicted model ( $C f$. Figure 6) does not fit the experimental one, we can assume that as for carbon fibers, hot pressing tends to orientate the carbon nanotubes inside the copper matrix. Assuming that, carbon nanotubes are randomly dispersed in a plane perpendicular to the axis of compression, the input thermal conductivity of CNT in the model of Maxwell-Garnett modified by Nan et al. has to be modified. Indeed, we have to take into account a global thermal conductivity of CNT (close to $1500 \mathrm{~W} \cdot \mathrm{m}^{-1} \cdot \mathrm{K}^{-1}$ ) which is more or less equal to the average of the thermal conductivity of the tube in the main axis $\left(3000 \mathrm{~W} \cdot \mathrm{m}^{-1} \cdot \mathrm{K}^{-1}\right)$ and the thermal conductivity of the tube perpendicular to the main axis $\left(10 \mathrm{~W} \cdot \mathrm{m}^{-1} \cdot \mathrm{K}^{-1}\right)$. Figure 7 shows experimental results in both direction (perpendicular and parallel to the axis of compression) and theoretical results $\left(K_{11}^{*}\right.$ and $\left.K_{33}^{*}\right)$. Theoretical results are plotted for CNT with a thermal conductivity of $3000 \mathrm{~W} \cdot \mathrm{m}^{-1} \cdot \mathrm{K}^{-1}$ (thermal conductivity along the main axis of the tube) and with a thermal conductivity of $1500 \mathrm{~W} \cdot \mathrm{m}^{-1} \cdot \mathrm{K}^{-1}$ (average thermal conductivity in the parallel and perpendicular direction of the tube).

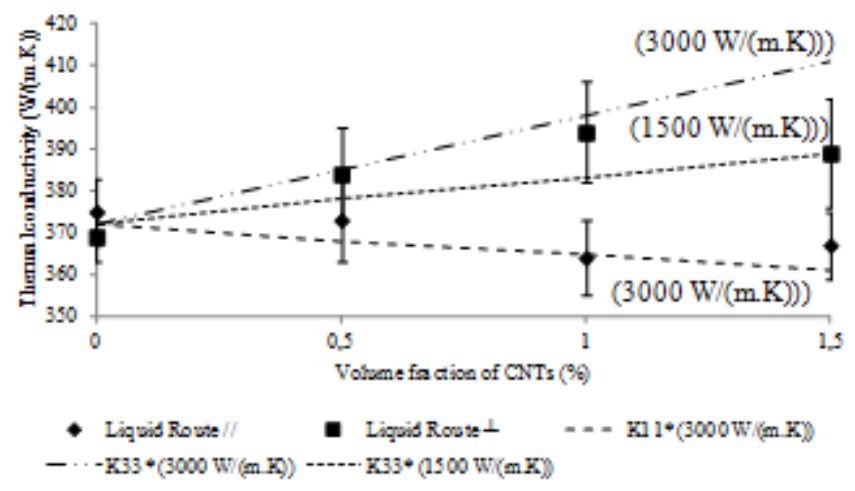

Figure 7. Thermal conductivity of Cu-CNTs composite materials in the direction parallel (Liquid Route (par)) and perpendicular (Liquid Route (per)) to the axis of compression and predicted results ( $K_{11}^{*}$ and $K_{33}^{*}$ ) for $\left\langle\cos ^{2} \theta\right\rangle=1$ and $\mathrm{K}_{\mathrm{CNT}}=3000 \mathrm{~W} \cdot \mathrm{m}^{-1} \cdot \mathrm{K}^{-1}$ and $\mathrm{K}_{\mathrm{CNT}}=1500 \mathrm{~W} \cdot \mathrm{m}^{-1} \cdot \mathrm{K}^{-1}$. 
Figure 7 clearly shows that the perpendicular thermal conductivity values measured are in good agreement with the $K_{33}^{*}$ plots obtained for $\mathrm{K}_{\mathrm{CNT}}=1500 \mathrm{~W} \cdot \mathrm{m}^{-1} \cdot \mathrm{K}^{-1}$. Therefore, taking into account the experimental thermal conductivity values measured in our $\mathrm{Cu}-\mathrm{CNTs}$ materials and the predicted ones calculated with the Maxwell-Garnett model modified by Nan et al., we can easily claimed that: i) CNT are well-dispersed into the copper matrix with the liquid route mixing process and ii) the uni-axial hot pressing process tends to randomly disperse the nanotubes in a plane perpendicular to the axis of compression leading to thermal conductivity measured values greater than our copper reference.

\section{Conclusions}

The concept of $\mathrm{Cu}-\mathrm{CNTs}$ composite materials with enhanced thermal properties compared with the pure matrix has been demonstrated. Indeed, an increase of the thermal properties of $+7 \%$ for a carbon nanotube content of $1 \%$ has been measured. The novelty of this work is to present a fabrication process of $\mathrm{Cu}-\mathrm{CNTs}$ composite material without using acid-treated carbon nanotubes. The non-functionalization process of the reinforcements enables to keep the physical properties of the nanotubes. Moreover, we demonstrate that the use of a theoretical model like Maxwell-Garnett modified by Nan et al. is an indirect method to quantify the dispersion of reinforcements into the matrix and for concentrations under the percolation threshold. The comparison of experimental thermal conductivity values with the predicted ones is an alternatively way to the microscope observations. MG model show that better enhancements of the thermal properties can be expected by aligning the tube in the matrix and a new process, different than uni-axial hot pressing, has to be developed. Moreover, the fabrication of $\mathrm{Cu}-\mathrm{CNTs}$ composite materials showing better thermal properties than the pure matrix may find some applications in the microelectronics field. Indeed, the miniaturization of power devices leads to an increase of the heat which has to be dissipated. Adding a composite material which exhibits a better thermal conductivity could help in this way.

\section{Acknowledgements}

The authors would like to thank BPI France, Aerospace Valley, Minalogic, I-Trans, Direction Générale de l'Armement and the region Nord-Pas de Calais for funding the $\mathrm{MC}+$ program.

\section{Appendix}

This appendix presents the details for the calculations in the model of Maxwell-Garnett modified by Nan et al.

$$
K_{11}^{*}=K_{22}^{*}=K_{m} \frac{2+f\left[\beta_{11}\left(1-L_{11}\right)\left(1+\left\langle\cos ^{2} \theta\right\rangle\right)+\beta_{33}\left(1-L_{33}\right)\left(1-\left\langle\cos ^{2} \theta\right\rangle\right)\right]}{2-f\left[\beta_{11} L_{11}\left(1+\left\langle\cos ^{2} \theta\right\rangle\right)+\beta_{33} L_{33}\left(1-\left\langle\cos ^{2} \theta\right\rangle\right)\right]}
$$

$$
K_{33}^{*}=K_{m} \frac{1+f\left[\beta_{11}\left(1-L_{11}\right)\left(1-\left\langle\cos ^{2} \theta\right\rangle\right)+\beta_{33}\left(1-L_{33}\right)\left(\left\langle\cos ^{2} \theta\right\rangle\right)\right]}{1-f\left[\beta_{11} L_{11}\left(1-\left\langle\cos ^{2} \theta\right\rangle\right)+\beta_{33} L_{33}\left(\left\langle\cos ^{2} \theta\right\rangle\right)\right]}
$$

Where $K_{11}^{*}, K_{22}^{*}$ and $K_{33}^{*}$ represent the effective thermal conductivity of the composite along the $X_{1}^{*}, \quad X_{2}^{*}$ and $X_{3}^{*}$ axis respectively.

$$
\begin{gathered}
L_{11}=\frac{p^{2}}{2\left(p^{2}-1\right)}-\frac{p}{2\left(p^{2}-1\right)^{3 / 2}} \cosh ^{-1} p \\
L_{33}=1-2 L_{11}
\end{gathered}
$$

Where $L_{i i}$ are well-known geometrical factors dependent on the particle shape. In our case $L_{11}=1 / 2$ and $L_{33}=0$.

$$
p=l / d
$$

Where $p$ is the aspect ratio of the nanotube, 1 is the length (in $\mathrm{nm}$ ) and $\mathrm{d}$ is the diameter (in $\mathrm{nm}$ ) of the nanotube. $\beta_{i i}$ is expressed with the following formula:

$$
\begin{aligned}
\beta_{i i} & =\frac{K_{i i}^{c}-K_{m}}{K_{m}+L_{i i}\left(K_{i i}^{c}-K_{m}\right)} \\
K_{i i}^{c} & =\frac{K_{C N T}}{\left(1+\gamma L_{i i} \frac{K_{C N T}}{K_{m}}\right)} \\
\gamma & =\left(2+\frac{1}{p}\right) * \frac{R_{t h} K_{m}}{d}
\end{aligned}
$$

Where $K_{i i}^{c}$ is the equivalent thermal conductivity along the $X_{i}$ symmetric axis of the ellipsoidal composite unit cell, $K_{m}$ and $K_{C N T}$ are the thermal conductivity of the matrix and the carbon nanotube respectively and $R_{t h}$ is the interfacial thermal resistance between the nanotube and the copper matrix.

\section{REFERENCES}

[1] S. Iijima, "Helical microtubules of gaphitic carbon", Nature 354 (1991) 56-58.

[2] S.H. Park, P.R. Bandaru, "Improved mechanical properties of carbon nanotube/polymer composites through the use of carboxyl-epoxide functional group linkages", Polymer 51 (2010) 5071-5077. 
[3] C.M. Damian, S.A. Garea, E. Vasile, H. Iovu, "Covalent and non-covalent functionalized MWCNTs for improved thermo-mechanical properties of epoxy composites", Composites: Part B 43 (2012) 3507-3515.

[4] P. Bonnet, D. Sireude, B. Garnier, O. Chauvet, "Thermal properties and percolation in carbon nanotube-polymer composites", Applied Physics Letters 91 (2007) 201910.

[5] E. Kymakis, G. Amaratunga "Electrical properties of single-wall carbon nanotube polymer composite films", Journal of Applied Physics 99 (2006) 084302.

[6] S. Chakraborty, J. Pionteck, B. Krause, S. Banerjee, B. Voit, "Influence of different carbon nanotubes on the electrical and mechanical properties of melt mixed poly(ether sulfone)-multi walled carbon nanotube composites", Composites Science and Technology 72 (2012) 1933-1940.

[7] P. Pisitsak, R. Magarapahan, S. Jana, "Electrically conductive compounds of polycarbonate, liquid, crystalline polymer, and multiwalled carbon nanotubes", Journal of Nanomaterials 2012 (2012) 642080

[8] K. Chu, Q. Wu, C. Jia, X. Liang, J. Nie, W. Tian, G. Gai, H. Guo, "Fabrication and effective thermal conductivity of multi-walled carbon nanotubes reinforced $\mathrm{Cu}$ matrix composites for heat sink applications", Composites Science and Technology 70 (2010) 298-304.

[9] A.K. Shukla, N. Nayan, S.V.S.N. Murty, S.C. Sharma, P. Chandran, S.R. Bakshi, K.M. George, "Processing of copper-carbon nanotube composites by vacuum hot pressing technique", Materials Science \& Engineering A 560 (2013) 365-371.

[10] S. Cho, K. Takagi, H. Kwon, D. Seo, K. Ogawa, K. Kikuchi, A. Kawasaki, "Multi-walled carbon nanotube-reinforced copper nanocomposite coating fabricated by low-pressure cold spray process", Surface and Coating Technology 206 (2012) 3488-3494.

[11] S. Cho, K. Kikuchi, T. Miyazaki, K. Takagi, A. Kawasaki, T. Tsukada, "Multiwalled carbon nanotubes as a contributing reinforcement phase for the improvement of thermal conductivity in copper matrix composite", Scripta Materialia 63 (2010) 375-378.

[12] K. Chu, Q. Wu, C. Jia, X. Liang, J. Nie, W. Tian, G. Gai, H. Guo "Fabrication and effective thermal conductivity of multi-walled carbon nanotubes reinforced $\mathrm{Cu}$ matrix composites for heat sink applications", Composites Science and Technology 70 (2010) 298-304.

[13] K. Chu, H. Guo, C. Jia, F. Yin, X. Zhang, X. Liang, H. Chen "Thermal properties of carbon nanotube-copper composites for thermal management applications", Nanoscale Research Letters (2010) 5:868-874.
[14] Q. Chen, "Carbon nanotubes reinforced metal composites", US Patent 2007/0036978A1, 2007.

[15] C-W. Nan, R. Birringer, D.R. Clarke, H. Gleiter, "Effective thermal conductivity of particulate composites with interfacial thermal resistance", Journal of Applied Physics 81 (1997) 6692-6699.

[16] S.T. Huxtable, D.G. Cahill, S. Shenogin, L. Xue, R. Ozisik, P. Barone, M. Usrey, M.S. Strano, G. Siddons, M. Shim, P. Keblinski, "Interfacial heat flow in carbon nanotube suspensions", Nature materials 2 (2003) 731-734.

[17] T.A. Saleh, "The influence of treatment temperature on the acidity of MWCNT oxidized by $\mathrm{HNO} 3$ or a mixture of $\mathrm{HNO}_{3} / \mathrm{H}_{2} \mathrm{SO}_{4}$ ", Applied Surface Science 257 (2011) 7746-7751.

[18] M. Estili, A. Kawasaki, H. Sakamoto, Y. Mekuchi, M. Kuno, T. Tsukada, "The homogeneous dispersion of surfactanless, slightly disordered, crystalline, multiwalled carbon nanotubes in a-alumina ceramics for structural reinforcements", Acta Materialia 56 (2008) 4070-4079.

[19] S.I. Cha, K.T. Kim, S.N. Arshad, C.B. Mo, S.H. Hong, "Extraordinary strengthening effect of carbon nanotubes in metal-matrix nanocomposites processed by molecular-level mixing”, Advanced Materials 17 (2005) 1377-1381.

[20] C. Ping, F. Li, Z. Jian, J. Wei, "Preparation of $\mathrm{Cu} / \mathrm{CNT}$ composite particles and catalytic performance on thermal decomposition of ammonium perchlorate", Propellants, Explosives, Pyrotechnics 31 (2006) 452-455.

[21] L. Xu, X. Chen, W. Pan, W. Li, Z. Yang, Y. Pu, "Electrostatic-assembly carbon nanotube-implanted copper composite spheres", Nanotechnology 18 (2007) 1-4.

[22] K.T. Kim, J. Eckert, S.B. Menzel, T. Gemming, S.H. Hong, "Grain refinement assisted strengthening of carbon nanotube reinforced copper matrix nanocomposites", Applied Physics Letters 92 (2008) 121901.

[23] I. Firkowska, A. Boden, A-M. Vogt, S. Reich, "Effect of carbon nanotube surface modification on thermal properties of copper-CNT composites", Journal of Materials Chemistry 21 (2011) 17541-17546.

[24] F. Cernuschi, L. Lorenzoni, P. Bianchi, A. Figari, "The effects of sample surface treatments on laser flash thermal diffusivity measurements", Infrared Physics \& Technology 43 (2002) 133-138.

[25] C-W. Nan, Z. Shi, Y. Lin, "A simple model for thermal conductivity of carbon nanotube-based composite", Chemical Physics Letters 375 (2003) 666-669.

[26] L. Berhan, A.M. Sastry, "Modeling percolation in high-aspect-ratio fiber systems. I. Soft-core versus hard-core models", Physical Review E 75 (2007) 041120-1-8. 